IMMUNOLOGY

\title{
Surprising role of cardiac macrophages in heart electrical conduction
}

Many studies have demonstrated that macrophages have multiple functions in different organs and tissues beyond their classical phagocytic activity. However, the specific functions of heart-resident macrophages in the steady state remained unclear. A new study published in Cell now shows that cardiac macrophages are involved in cardiac electrical conduction. "Macrophage depletion leads to atrioventricular [AV] block, and macrophages that are connected to cardiomyocytes via gap junctions rhythmically depolarize," explains Matthias Nahrendorf, corresponding author of the study. "Both results were completely shocking," he adds.

Nahrendorf and colleagues analysed murine hearts as well as human hearts obtained from autopsies of patients with no known cardiac conduction disease. The investigators used optical clearing followed by confocal fluorescence microscopy imaging of whole AV nodes to assess the intraorgan distribution and morphology of cardiac

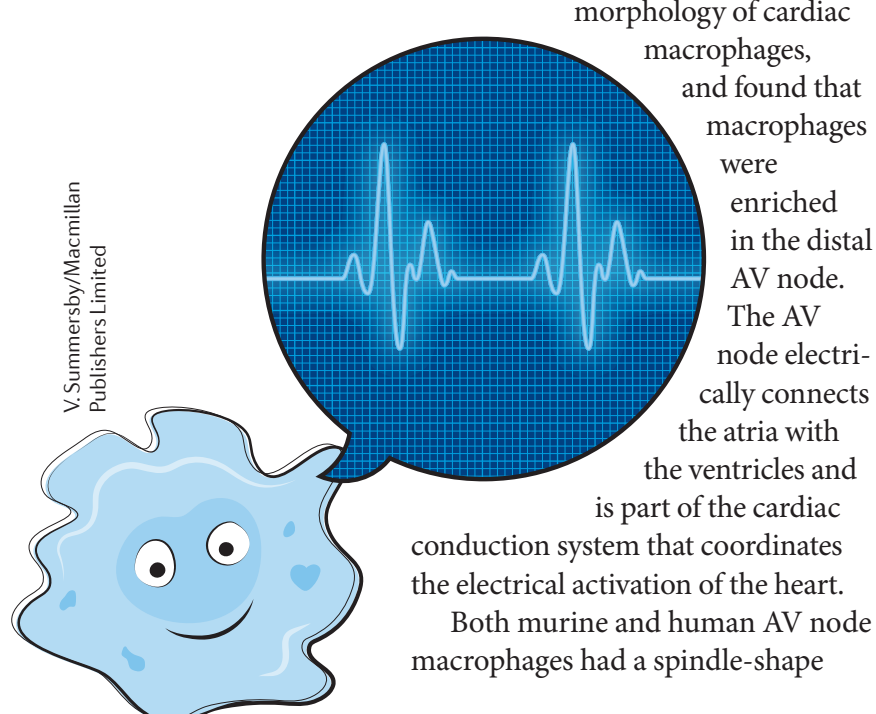

morphology with far-reaching protrusions. Conducting cardiomyocytes frequently interspersed with macrophages, particularly in the nodal or AV bundle. Single-cell RNA-sequencing showed that the gene-expression profile of murine AV node macrophages was similar to that of ventricular macrophages. Interestingly, both cell types expressed genes coding for proteins involved in electrical conduction.

Nahrendorf and colleagues further demonstrated that connexin-43 (Cx43)-containing gap junctions connect macrophages with conducting cardiomyocytes in the distal AV node. Previous studies have shown that $\mathrm{Cx} 43$-containing gap junctions not only electrically couple cardiomyocytes, but also electrotonically couple heterotypic cells. Indeed, using a series of in vitro models, the investigators demonstrate that cardiac macrophages electrotonically communicate with coupled cardiomyocytes. Macrophages connected to spontaneously beating cardiomyocytes via Cx43-containing gap junctions depolarized in synchrony with the coupled cardiomyocytes and had a more negative resting membrane potential than that of solitary macrophages. Interestingly, cardiac macrophages in turn altered the electrophysiological properties of the coupled cardiomyocytes. Macrophages rendered the resting membrane potential of coupled cardiomyocytes more positive, which was reversed by pharmacological blockade of Cx43. In addition, computational modelling indicated that cardiac macrophages accelerate cardiomyocyte repolarization.
A optogenetic approach enabled Nahrendorf and colleagues to assess this macrophage-cardiomyocyte communication directly in the AV node of mouse isolated hearts. The investigators generated a mouse model with tamoxifen-inducible expression of the photoactivatable channelrhodopsin 2 specifically in macrophages to control their membrane potential. Photostimulation of hearts isolated from these mice, which induced a conformational change that opened the cation channelrhodopsin 2 channel and depolarized the macrophages, improved AV node conduction. By contrast, both conditional deletion of $C \times 43$ in macrophages and congenital lack of macrophages delayed AV node conduction in mice. Furthermore, acute macrophage depletion in mice, achieved using the $C d 11 b^{D T R}$ mouse model, induced AV block.

Nahrendorf and colleagues conclude that cardiac macrophages can couple to conducting cardiomyocytes via $\mathrm{Cx} 43$-containing gap junctions. This coupling leads to cyclical depolarization of macrophages and modulates the electrical activity of cardiomyocytes. Enhanced macrophage-cardiomyocyte interaction improves normal AV nodal conduction, whereas reduced interaction leads to aberrant $\mathrm{AV}$ node conduction. Nahrendorf and colleagues are planning to address how macrophages are involved in conduction disorders such as atrial or ventricular fibrillation.

Irene Fernández-Ruiz

ORIGINAL ARTICLE Hulsmans, M. et al.

Macrophages facilitate electrical conduction in the heart. Cell 169, 510-522 (2017) 